\title{
التظم مفتوحة المصدر لإدارة أعمال المؤتمرات
}

\author{
عرض \\ د. إهداء صلاح ناجي \\ مدرس المكتبات وعلم المعلومات \\ قسم المكتبات والوثائق وتقنية المعلومات \\ كلية الآداب - جامعة القاهرة
}

0/تمصيد

تعد أعحال المؤتمرات العمود الفقري للاتصال العلمي، وأحد أهم منافذ الاتصال العلمي؛ حيث

إنه ليس كل ما ينشر من مخرجات البحث العلمي يصدر في شكل أطروحات جامعية أو كتب أو مقالات دوريات، ولكن تحظى المؤتمرات العلمية بنصيب من تلك المخرجات، فهي تعد أحد المصادر الرئيسية للمعلومات في مجال ما، وعلى الرغم من أهميتها، والزيادة الهائلة في كم ما يعقد سنويًا منها، إلا أن هذا تهات

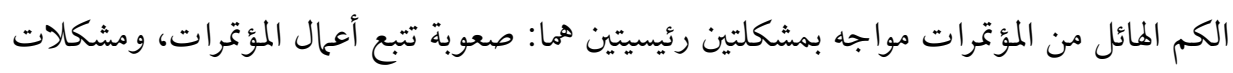

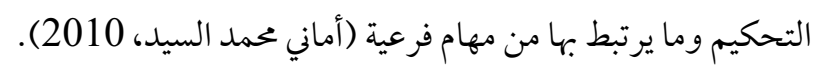

وتعمل المؤسسات الأكاديمية في جميع أنحاء العالم على تنظيم المؤتمرات سنويًا، تقديرًا لدورها في متئي

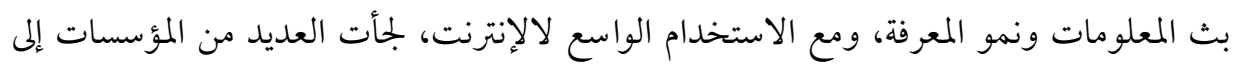

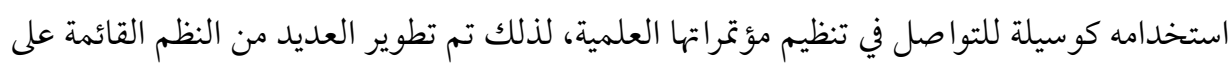
الويب لإدارة المؤتمرات العلمية، وتؤدي هذه النظم مجموعة من الوظائف الأساسية منها: تقديم

1- إهداء صلاح ناجي (2018). النظم مفتوحة المصدر لإدارة أعمال المؤتمرات العلمية: دراسة تقييمية مع اقتراح

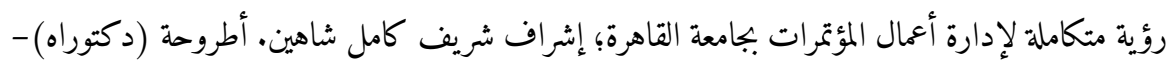
جامعة القاهرة، كلية الآداب، قسم المكتبات والوثائق وتقنية المعلومات. 
البحوث، وتحكيمها، وإخطار الباحثين بقبول، أو رفض أبحاثهم، وتسجيل المشاركين، وحفظ أعمال المؤتمرات و إتاحتها (Huang, 2009).

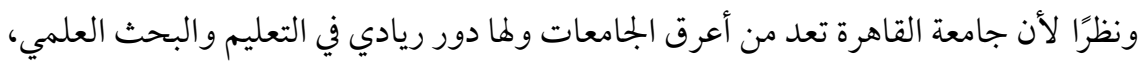

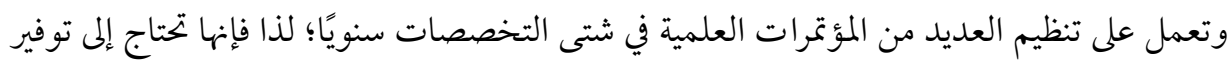

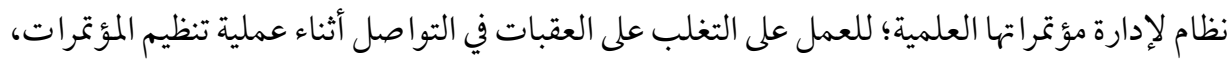

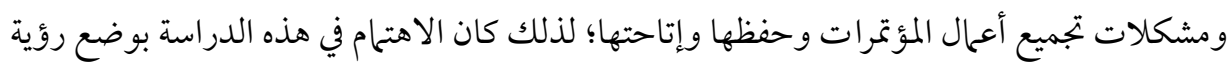

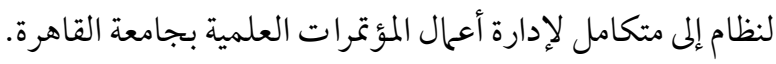

\section{1 /مشكلة الدراسة وأهميتها}

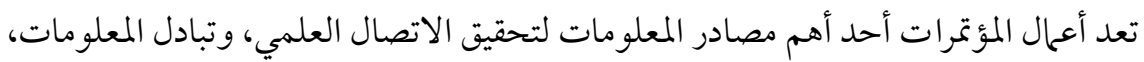

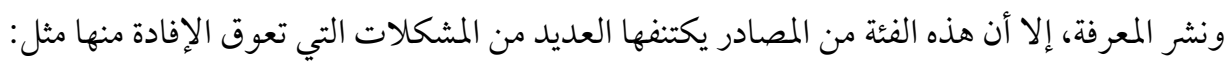

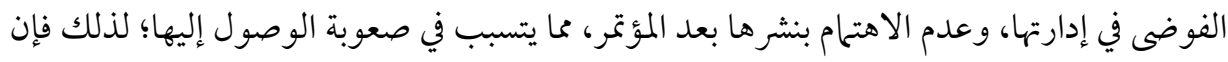

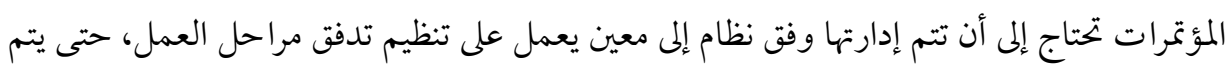
نشرها وإتاحتها.

وفى العقد الأخير ظهرت العديد من البريجيات، التي كان لها أثر كبير في تنظيم مراحل إدارة المؤتمرات العلمية، بداية من الإعلان عن المؤتمر، مرورًا بإرسال البحوث من جانب البعات الباحثين المشاركين، وإدارة عملية التحكيم، وتسجيل المشاركين، حتى نشر أعمال المؤتمر وإتاحتها.

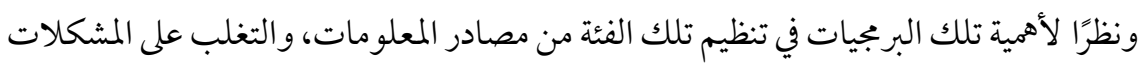

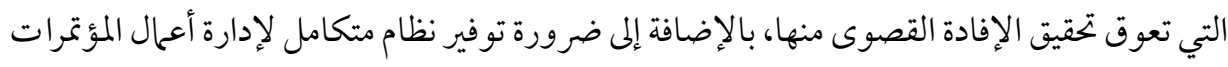

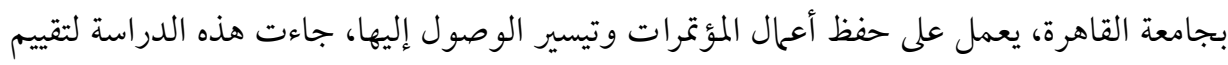

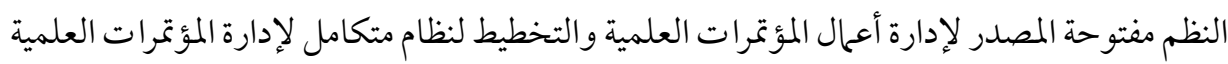

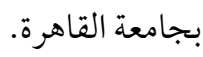

لقد أوصت دراسة (أماني محمد السيد، 2010) بضرورة قيام المكتبات الجامعية بتبني نظامًا لإدارة

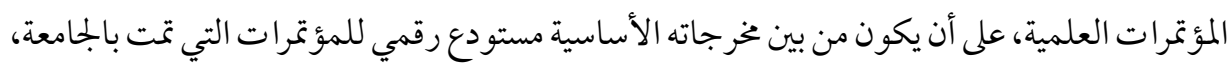

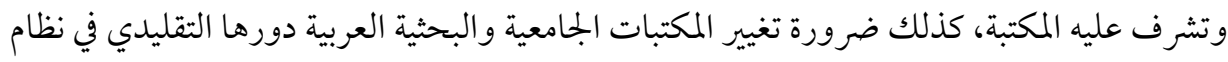


الاتصال العلمي من خلال تبني مبادرات تسهم في تطبيق نماذج غير تقليدية للنشر داخل نطاق الجامعة؛ اعتمادًا على مخرجاتها من البحوث العلمية ونظم النشر الرقمي.

\section{/ أهداف الدراسة}

تهدف هذه الدراسة - في المقام الأول - إلى وضع رؤية متكاملة لنظام لإدارة أعمال المؤتمرات

العلمية بجامعة القاهرة إلكترونيًا، وذلك من خلال تحقيق مجموعة من الأهداف الفرعية التالية:

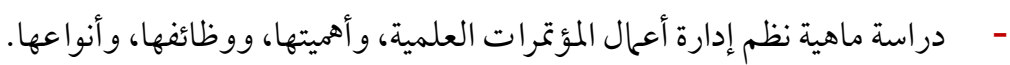

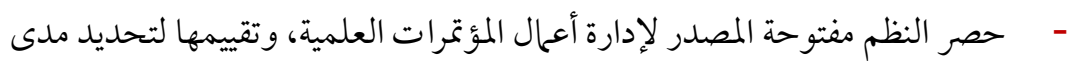

$$
\text { كفاءتها لإدارة المؤتمرات العلمية. }
$$

- دراسة تطبيقات نظم إدارة أعمال المؤتمرات العلمية بالجامعات والمكتبات عربيًا

وعالميًا.

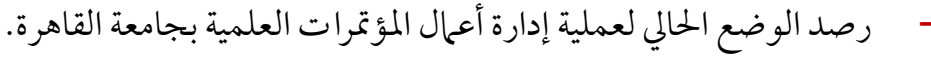

-

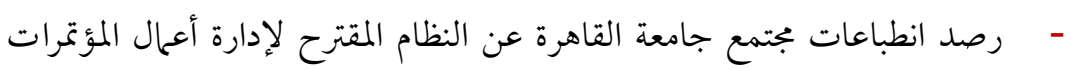

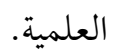

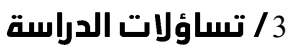

في ضوء الأهداف التي تسعى الدراسة لتحقيقها، فإن التساؤلات التي حاولت الإجابة عليها هي:

-

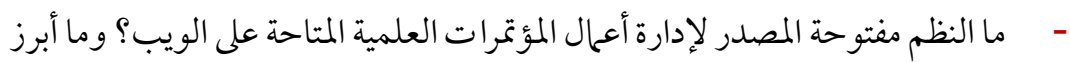

ملامعها وعناصر القوة والضعف فيها؟

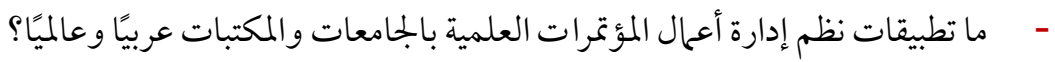

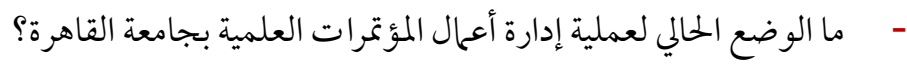

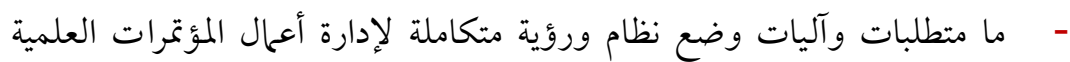

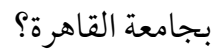

- ما مدى فاعلية وسهولة استخدام نظام إدارة أعلال المؤتمرات العلمية المقترح بجامعة

القاهرة؟ 


\section{4/ حدود الدراسة}

تتناول الدراسة النظم مفتوحة المصدر لإدارة أعمال المؤتمرات العلمية، في ضوء الحدود التالية:

الحدود الموضوعية

OCS, HOTCRP دراسة النظم مفتوحة المصدر للإدارة المؤتمرات العلمية ومن أشهرها: Conftool, Openconf, Easychair اقتراح رؤية متكاملة لإدارة المؤتمرات العلمية بجامعة القاهرة.

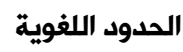

تتناول الدراسة النظم مفتوحة المصدر لإدارة أعمال المؤتمرات العلمية على أن يكون لها واجهة واحدة على الأقل باللغة الإنجليزية.

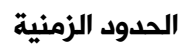

تغطى الدراسة تحليل النظم مفتوحة المصدر لإدارة أعلال المؤتمرات، وتقييمها منذ بداية ظهورها

في متصف التسعينيات حتى (يناير 2017)، وهو تاريخ إنهاء ملاحظة وتقييم تلك النظم.

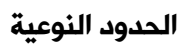

تشمل الدراسة النظم مفتوحة المصدر لإدارة أعمال المؤتمرات العلمية، التي تعمل على حفظ تلك

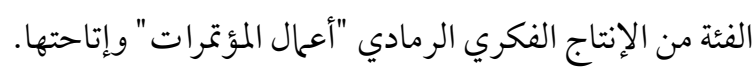

5 / منهج الدراسة وأدوات جمع البيانات

1/5منهج الدراسة

في ضوء أهداف الدراسة وحدودها، فإنها اعتمدت على:

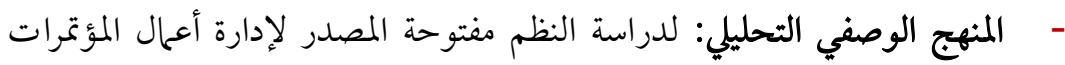

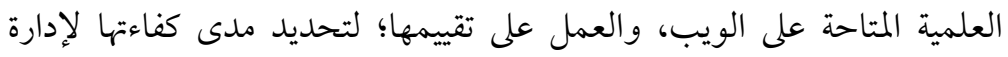

$$
\text { المؤتمرات العلمية. }
$$

- أسلوب تحليل وتصميم النظم: من أجل تصميم نظام لإدارة أعمال المؤتمرات العلمية

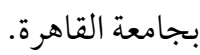


- أسلوب البحث التقييمي: Evaluative Research لتطبيق نظام إدارة أعمال المؤتمرات المقترح، ومعرفة مدى فاعليته وقابلية استخدامه من قبل بجمو عة من المستخدمين.

2/5 أدوات جمع البيانات

اعتمدت الباحثة - في جمع البيانات اللازمة للدراسة - على بجموعة من الأدوات، والتي تمثلت

\section{قائمة مراجعة Checklist}

وهي عبارة عن قائمة مراجعة كمية تضم (62) عنصرًا ، تهدف إلى تقييم آليات عمل تلك النظم،

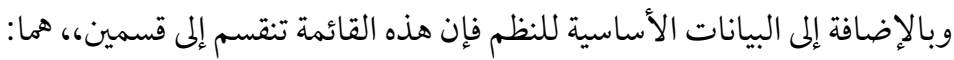

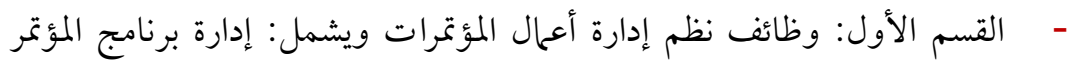

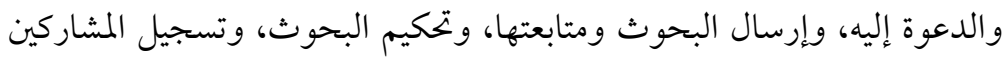
بالمؤتر، ونشر أعمال المؤتمر وإتاحتها.

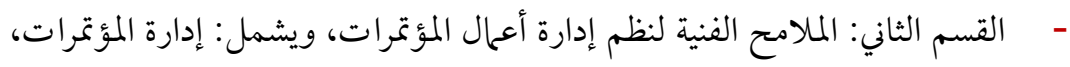

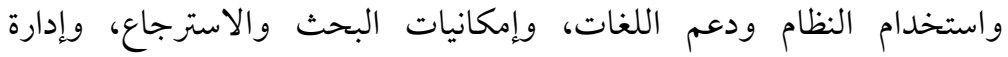

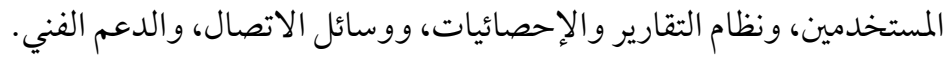
وقد تم تحديد عناصر قائمة المراجعة وتحكيمها من خلال المراحل التالية:

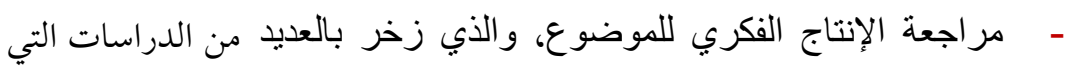
استطاعت منها الباحثة إعداد قائمة المراجعة.

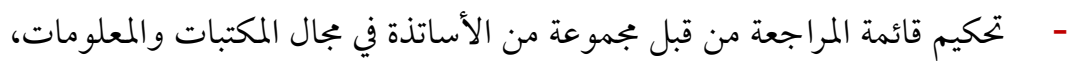
وقد تمثلت آراء السادة الأساتذة في الآتي:

$$
\begin{aligned}
& \text { - - تغيير صياغة بعض العبارات. - } \\
& \text { - - }
\end{aligned}
$$

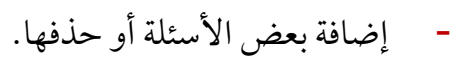

$$
\begin{aligned}
& \text { - - مديلات في شكل القائمة وتنسيقها. }
\end{aligned}
$$

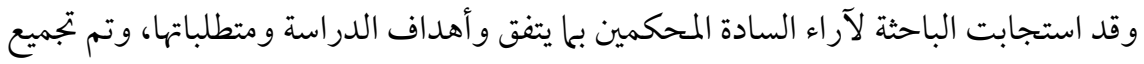

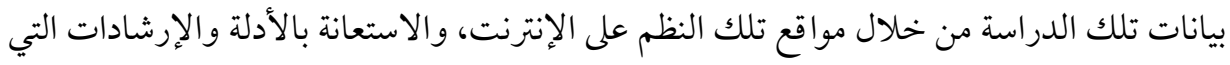


توفرها بعض هذه النظم، وتلك النظم هي (Cyberchair - easychair - MyReview sy)، والدخول

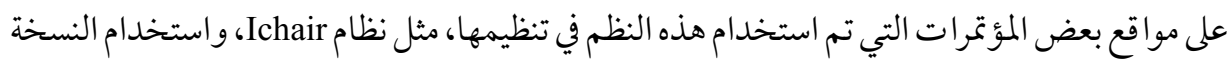

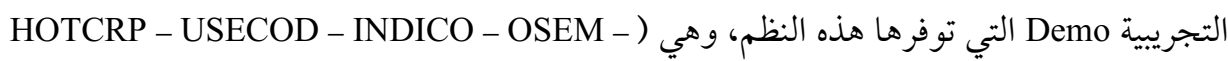
(ConfTool )، وتنصيب وتجريب بعض هذه النظم لغرض تقييمها، وهي (OCS - OpenConf)، وأيضًا

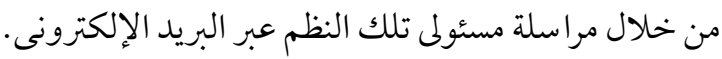

\section{المقابلة الشخصية}

وذلك بإجراء مقابلة مع وكلاء الكليات لشئون خدمة المجتمع وتنمية البيئة وعدد من أعضاء

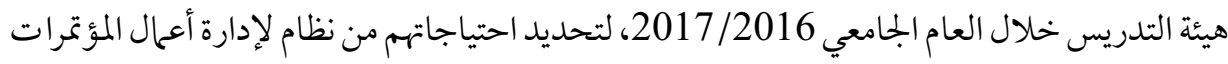

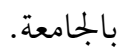

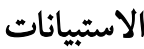

قامت الباحثة بتطبيق مقياسين على أعضاء هيئة التدريس بجامعة القاهرة لاختبار قابلية النظام للاستخدام، كالتالي:

- الأول: مقياس قابلية استخدام النظام: وهو يتكون من 10 جمل استبيانية، وكل جملة

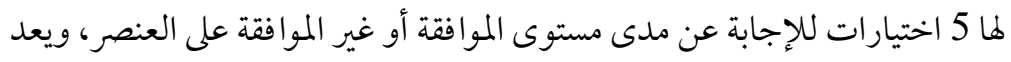
من أكثر المقاييس استخدامًا لقياس قابلية الاستخدام.

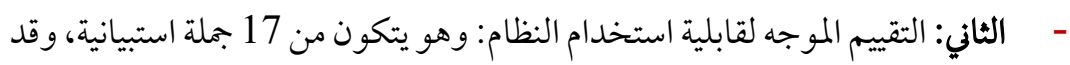

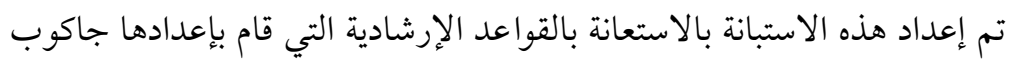

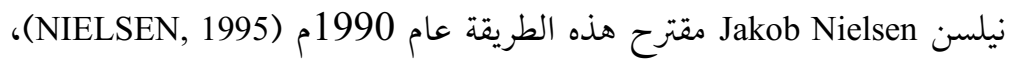

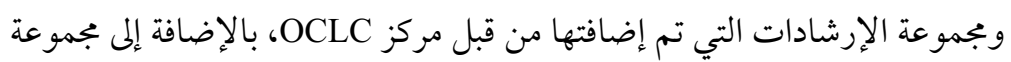

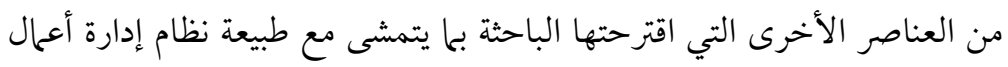

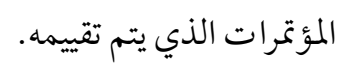

3/5 مجتمع وعينة الدراسة

$$
\text { ينقسم مجتمع الدراسة وفقًا لطبيعة الدراسة إلى قسمين: }
$$

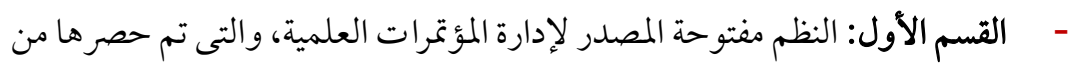

خلال الاعتماد على محر كات البحث، و كذلك من خلال الإل الاطلاع على الإنتاج الفكري 
العربي والأجنبي حول الموضوع، وبلغ عدد تلك النظم (21) نظامًا حتى يناير

2017، وتم العمل على تقييمها جميعها باستثناء تخسة نظم منها.

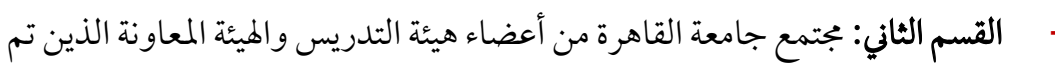

إجراء اختبار قابلية الاستخدام عليهم، وتم اختيار عينة الدراسة، كالتالي:

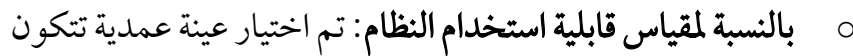

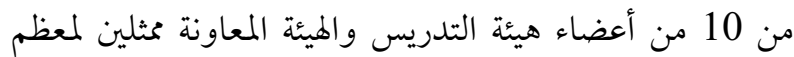

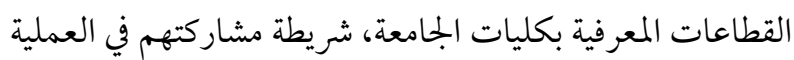

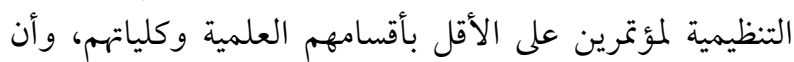

تكون تلك المؤتمرات تم عقدها في الثلاث سنوات الأخيرة.

o بالنسبة للتقييم الموجه لقابلية استخدام النظام: تم اختيار عينة

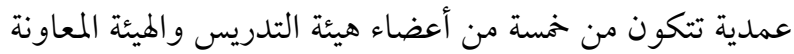

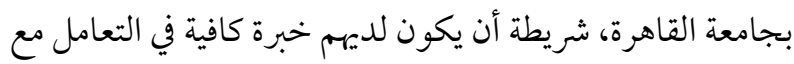

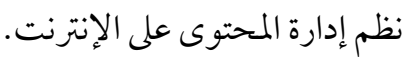

6/ فصول الدراسة

فضلًا عن المقدمة، انتظمت محتويات المادة العلمية بهذه الدراسة في أربعة فصول متعاقبة عكست

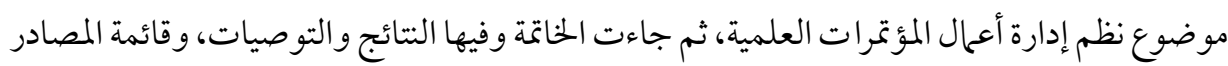

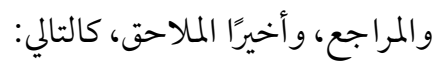

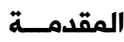

تشتمل على تهيد، وتعريف بالموضوع، وأهميته، والهدف منه، وتساؤلات الدراسة، ثم الحدود

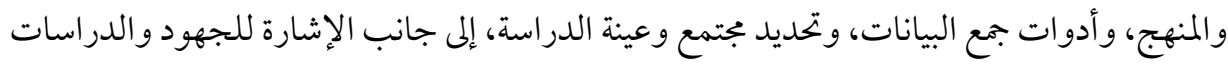
السابقة على المستويين العربي والأجنبي التي ترتبط بتلك والميات والدراسة.

الفصل الأول: نظم إدارة أعمال المؤتمرات العلمية: إطار نظري

يعرض هذا الفصل الإطار النظري للدراسة، وذلك من خلال إلقاء الضوء على المؤتمرات

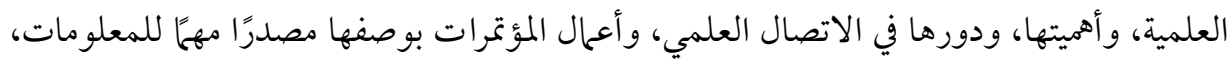

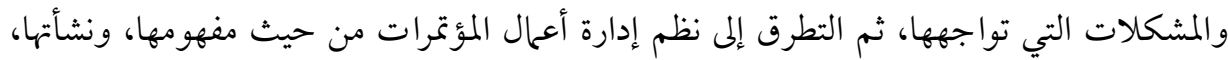


وتطورها، وأهميتها، ووظائفها، وبنيتها على الإنترنت، وخطوات تنظيم المؤترات العلمية باستخدام نظم إدارة المؤتمرات، ودورة تدفق العمل داخلها.

\section{الفصل الثاني: تطبيقات نظم إدارة أعمال المؤتمرات العلمية ومجالات تطويرها} يُعنى هذا الفصل بدراسة تطبيقات نظم إدارة أعمال المؤتمرات العلمية، ومجالات تطويرها من

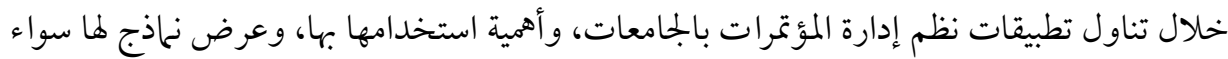

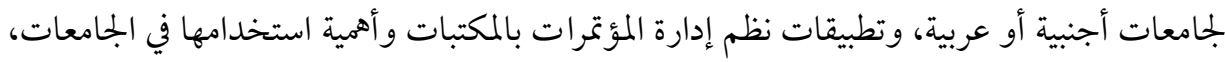

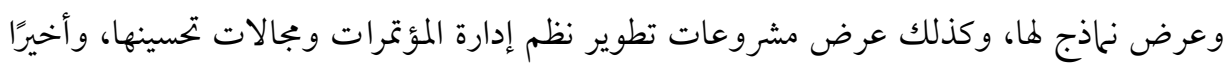
عرض نماذج لنظم إدارة مؤتمرات عربية.

الفصل الثالث: تقييم النظم مفتوحة المصدر لإدارة أعمال المؤتمرات العلمية

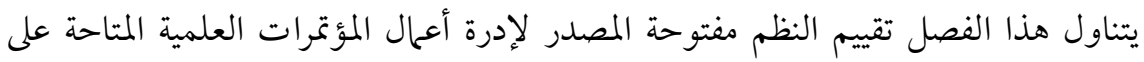

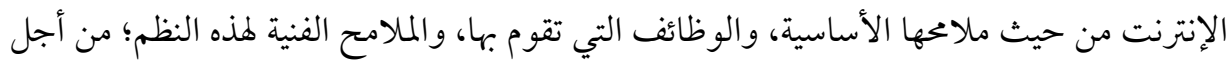
الخروج بنظام يصلح تطبيقه داخل المحيط الأكاديمي لجامعة القاهرة

\section{الفصل الرابع: نحو نظام إلى متكامل لإدارة المؤتمرات العلمية بجامعة القاهرة}

جاء هذا الفصل ليهدف إلى التخطيط لنظام إدارة المؤتمرات بجامعة القاهرة، وذلك بعد رصد

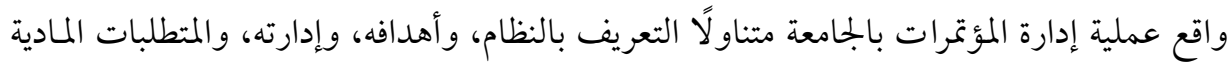

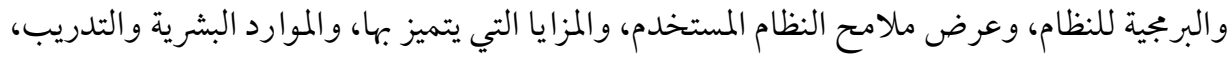
ثم خطوات تصميم النظام واختبار صفحاته، واختبار قابلية استخدام النظام بعد إتاحة الأدلة الإرشادية

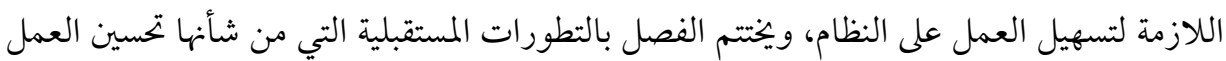
بالنظام.

\section{الملاحق}

ذُيلت الدراسة بخمسة ملاحق؛ خُصص الأول: قائمة مراجعة تقييم النظم مفتو حة المصدر لإدارة

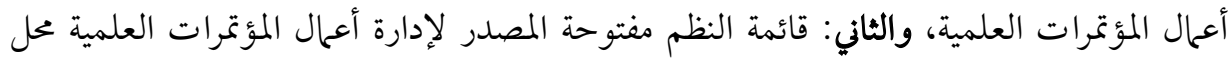

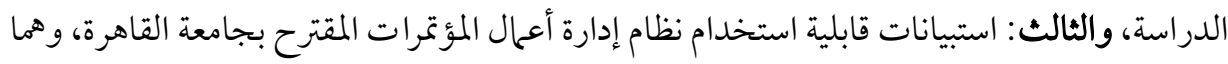

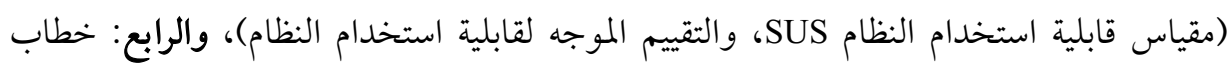


التوصية بإرسال أعمال المؤتمرات لإيداعها على المستودع الرقمي للجامعة، والخامس: أدلة نظام إدارة

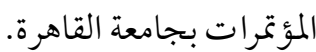

الخاتمة: النتائج والتوصيات.

جاءت نتائج البحث وتوصياته لتلخص ما انتهت إليه الباحثة في دراستها بمجموع فصوهلا

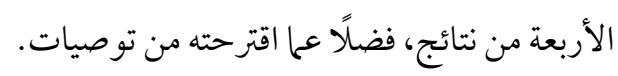

وقد توصلت الدراسة إلى مجموعة من النتائج والتوصيات يمكن إييازها فيمات يلى:

أولَا: التنائج

قادت هذه الدراسة إلى الإجابة عن مجموعة من التساؤلات التي ارتبطت بالهدف من إجرائها،

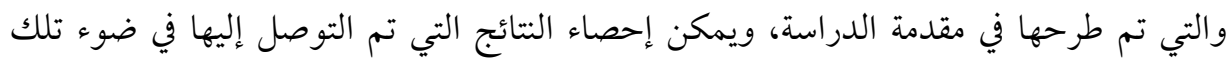

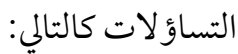

\section{ما النظم مفتوحة المصدر لإدارة المؤتمرات العلمية المتاحة على الويب؟ وما ابرز ملامصها}

وعناصر القوة والضعف فيها؟

بلغ عدد النظم مفتوحة المصدر لإدارة المؤتمرات العلمية 21 نظامًا (حتى يناير

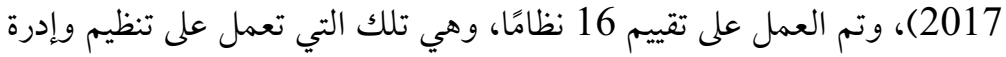

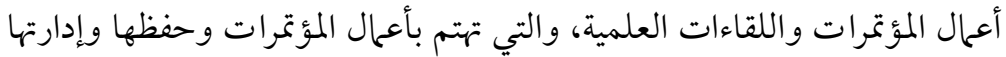
وإتاحة الوصول إليها بمستويات غختلفة.

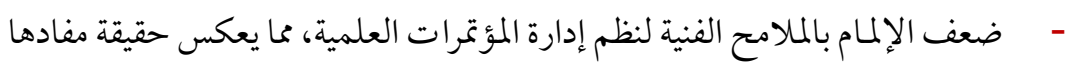

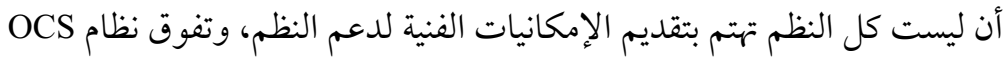
وفقًا لعناصرا لتقييم بقائمة المراجعة.

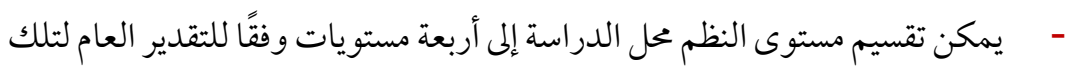

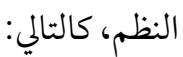

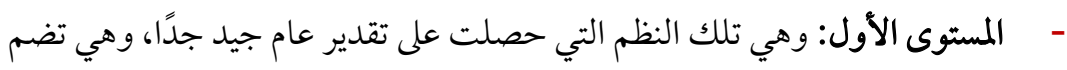

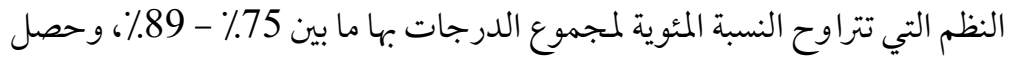
نظام OCS Oقط على هذا التقدير. 
- المستوى الثاني: وهي تلك النظم التي حصلت على تقدير عام جيد، وهي تضم النظم

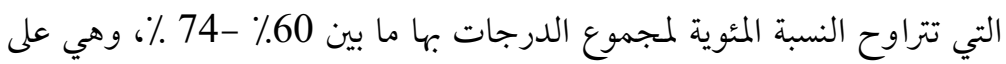
الترتيب USECOD - HOTCRP - Cyberchair - ConfTool - INDICO

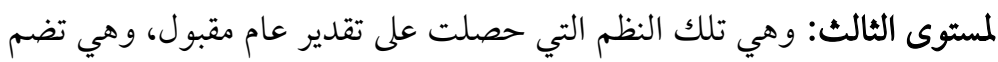

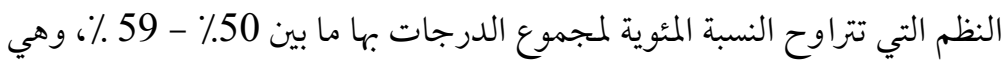

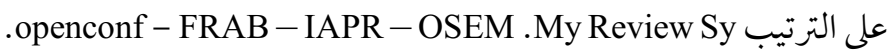

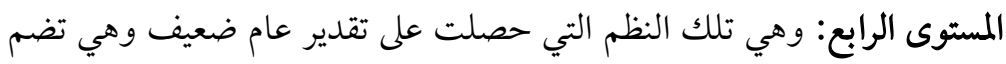

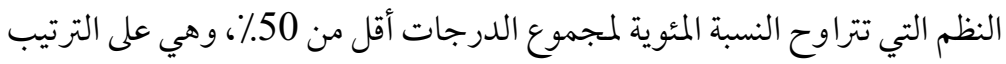
.CMS - Open Conference Ware - ichair - easychair - WCMT النظم التي تقوم عليها المؤسسات والمشروعات تعد الأقوى مثل OCS الذي تقوم

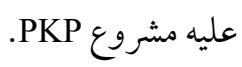

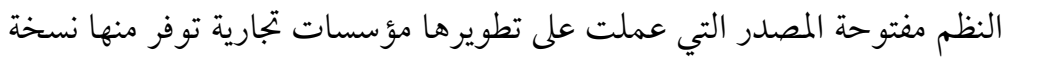

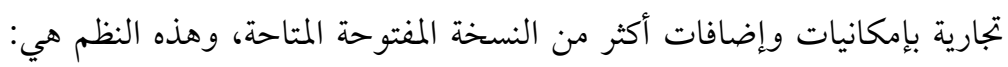
.openconf-ConfTool-HOTCRP-CyberChair

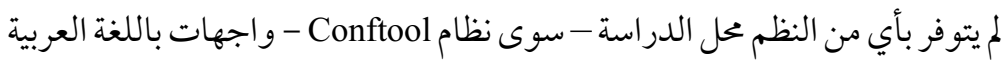

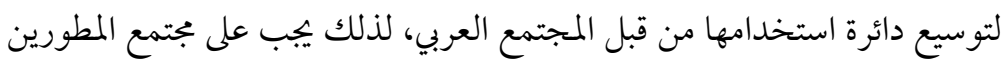

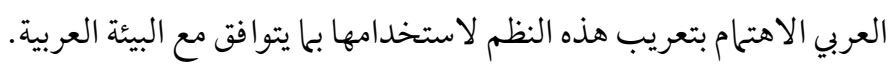

ما تطبيقات نظم إدارة اعمال المؤتمرات العلمية بالجامعات والمكتبات عربيًا وعالميًا ؟

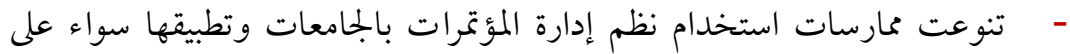

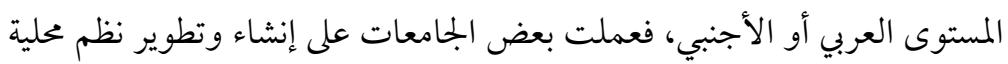

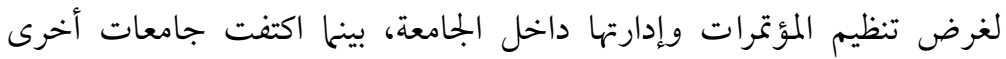
باستخدام نظم جاهزة سواء كانت مفتوحة المصدر أو تجارية لإدارة مؤتمراتها.

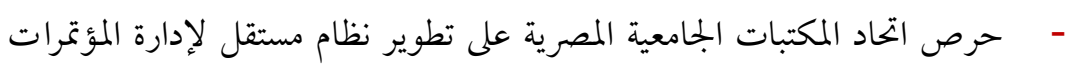

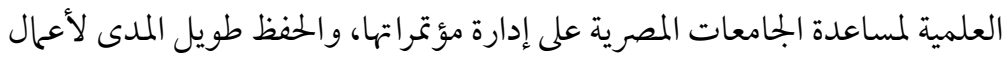

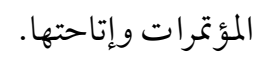




\section{ما الوضع الحالي لإدارة المؤتمرات العلمية بجامعة القاهرة؟}

- - عدم استخدام الكليات والأقسام العلمية أى نظام لإدارة مؤتمراتها فييا عدا كلية

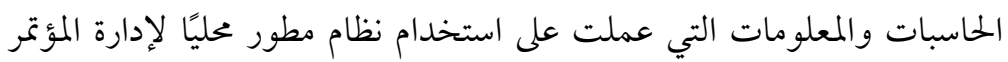

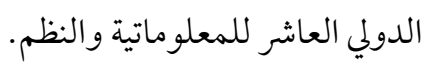

تعددت أوجه نشر أعلال المؤتمرات سواء من خلال الأقراص المليزرة أو طباعة كتاب

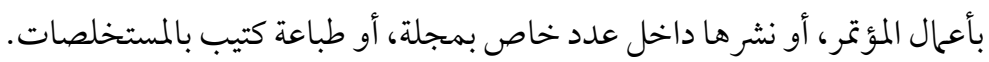

\section{ما متطلبات وآليات وضع نظام ورؤية متكاملة لإدارة اعمال المؤتمرات العلمية بجامعة القاهرة؟}

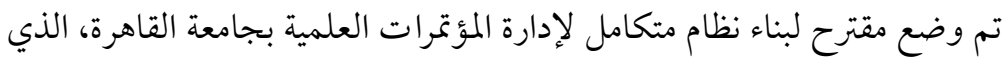

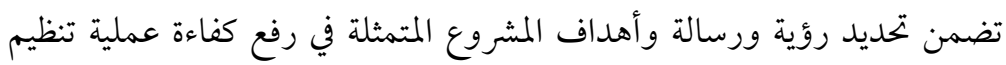

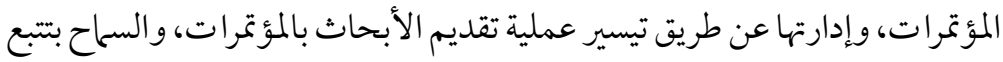

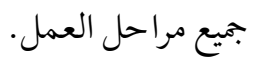

\section{ما مدى فاعلية وسهولة استخدام نظام إدارة اعمال المؤتمرات العلمية المقترح بجامعة القاهرة؛}

- أثمرت عملية تجريب نظام إدارة المؤتمرات العلمية بجامعة القاهرة وإجراء اختبار

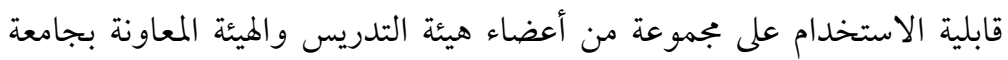

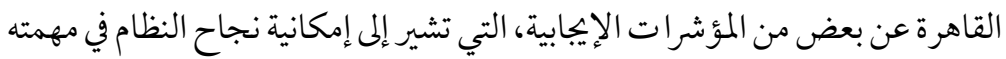

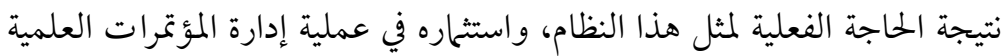

$$
\text { داخل بجتمع الجامعة. }
$$

يجتاج النظام كي ينجح إلى تدريب المستخدمين عليه، ولابد من توفير الدعم الفني اللازم لإنجاح النظام.

جاءت خطوات القيام بالعمليات داخل النظام مسلسلة، ومنطقية حسب مراحل تنظيم المؤتمرات؛ فالنظام ما هو إلا انعكاس لعملية تنظيم المؤتمرات، وما يرتبط بهات بهات الفيا

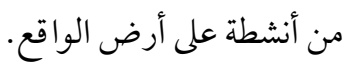

وأخيرًا يعد اختبار النظام نقطة البداية لتطبيقه على أرض الواعل الواقع والإفادة من

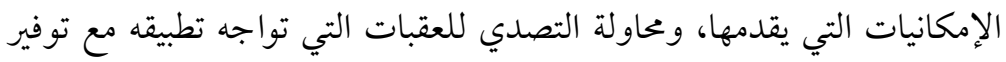

$$
\text { الدعم الفني والتدريب اللازم لاستخدام النظام. }
$$




\section{ثانيًا: التوصيات}

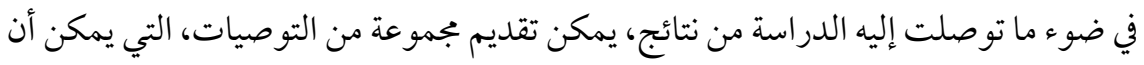

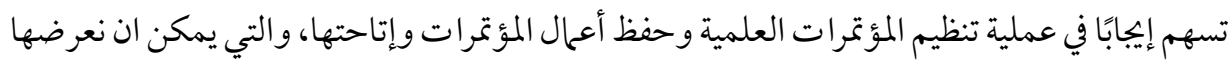
فيا يلي:

- العمل على استخدام نظم إدارة المؤتمرات العلمية في تنظيم المؤتمرات العلمية العربية واستثمارها، من أجل حفظ أعمال المؤتمرات وإتاحتها على الإنترنت.

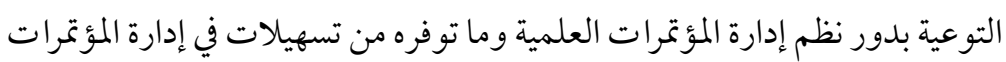

$$
\text { وتنظيمها، من أجل التشجيع على استخدامها. }
$$

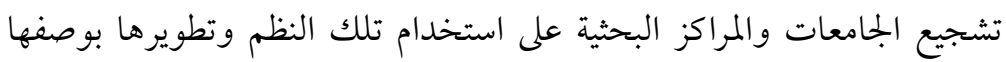

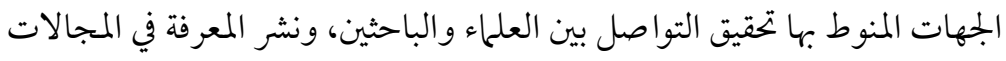

$$
\text { العلمية المختلفة. }
$$

الاهتمام من قبل المؤسسات الأكاديمية العربية بتعريب هذه النظم، وتوفير الأدلة الإدية

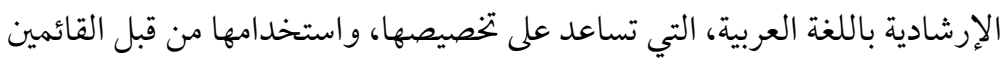

$$
\text { على تنظيم المؤتمرات العلمية. }
$$

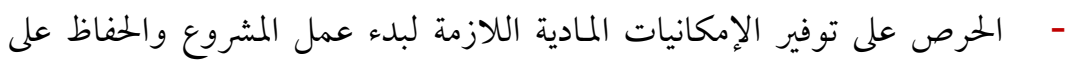

$$
\text { استمراره. }
$$

- عمل خطط تدريبية للتدريب على استخدام نظام إدارة المؤتمرات العلمية لأعضاء هيئة

$$
\text { التدريس، ومعاونيهم القائمين على تنظيم المؤتمرات العلمية. }
$$

\section{المصادر}

أماني محمد السيد. (2010). نظم إدارة المؤتمرات العلمية ودورها في تحكيم ونشر بكوث المؤتمرات: دراسة

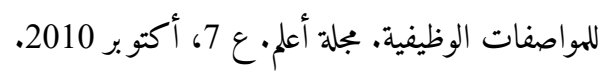

Huang, M. (2009). CONFSYS2: A Redesigned Web-based Multi-Conference Management System. Master

Thesis, Concordia University. Retrieved $\quad$ Oct $7, \quad 2015$, from https://spectrum.library.concordia.ca/976467/1/MR63115.pdf 